\title{
Multiparametric magnetic resonance imaging in the diagnosis of clinically significant prostate cancer: A call to action
}

\section{Bobby Shayegan, MD}

McMaster Institute of Urology, St. Joseph Healthcare, Hamilton, ON, Canada

Cite as: Shayegan B. Multiparametric magnetic resonance imaging in the diagnosis of clinically significant prostate cancer: A call to action. Can Urol Assoc J 2022;16(2):15. http://dx.doi. org/10.5489/cuaj.7766

See related CCO guideline on page 16

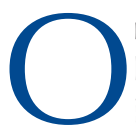
ntario Health's (formerly Cancer Care Ontario) Program in Evidence-Based Care recently published its long-awaited clinical guidance on the use of multiparametric magnetic resonance imaging (mpMRI) in the diagnosis of clinically significant prostate cancer (csPCa). The importance of this position paper lies principally in recommendations for biopsy-naive men, which follows the global shift towards upfront mpMRI to avoid unnecessary biopsies and over-detection of clinically insignificant prostate cancers.

There is no doubt that the evolution of high-quality mpMRI with standardized reporting has resulted in changes in the way prostate cancer patients are managed in Canada today. Much of the present experience in Canada lies in the use of $\mathrm{mpMRI}$ in patients with previously negative transrectal ultrasound-guided systematic biopsy (TRUS-SB) and ongoing clinical concern for a clinically significant cancer. Lessons learned from this experience mirror the published literature: high-resolution MR technology and radiologist experience are essential elements of a successful MR program in prostate cancer.

Detection of csPCa on mpMRI (whether biopsy-naive or not) requires the appropriate MRI technology. Most published studies have used 3T-MRI units for improved signalto-noise ratio and better spatial resolution (especially if not using endorectal coils). This is essential for identification of small lesions within anatomically complex structures.

In addition to technological requirements, an experienced radiology team is critical to reliable and consistent reporting. While it is reported that the performance of $\mathrm{mpMRI}$ is acceptable in detection of csPCa (with $8-24 \%$ having a false-negative report), this depends heavily on the experience of the radiologist. As pointed out in this article, national and regional development of quality assurance programs is critical to ensuring the success of such an endeavour.

Experience in detection and reporting of csPCa on mpMRI now spans over two decades. The benefits, however, cannot be entirely realized without accurate targeting of lesions of interest. This is likely the rate-limiting step in a broad rollout of such an initiative. While cognitive fusion can be performed without additional resources, TRUS-MRI fusion and in-bore MRI biopsy are likely to improve yield. The latter is more time-consuming and resource-intensive and unlikely to be broadly practiced. Irrespective of which fusion platform is used, there is a distinct paucity of such technologies in Canada.

The literature clearly supports the use of mpMRI in biopsy-naive men (as well as those with a previous negative TRUS-SB but ongoing clinical suspicion) for detection of csPCa and subsequent mpMRI-targeted biopsy (with or without concurrent TRUS-SB). The endorsement by the CUA of this clinical guidance by Ontario Health is an important, positive step for the Canadian urology community; however, the benefit to patients can only be realized with significant investment in the technology, training, and time.

At present, most hospitals do not have the required resources to ramp up $\mathrm{mpMRI}$ or offer fusion-based targeted biopsies. It is imperative that prostate cancer clinicians in leadership positions and hospital administrators recognize this unmet need and work towards bridging this gap quickly. It is unlikely that a few reference centers will be able to become regionalized centers for TRUS-MRI fusion biopsies given anticipated volumes of positive mpMRI in biopsy-naive men. As such, this report should also serve as a call to action for hospitals and stakeholders in prostate cancer.

Competing interests: Dr. Shayegan has been an advisory board member for Astellas, Bayer, and Janssen; and has received a research grant from Janssen.

Correspondence: Dr. Bobby Shayegan, McMaster Institute of Urology, St. Joseph Healthcare, Hamilton, ON, Canada; shayeb@mcmaster.ca 